\title{
A Model-Based Supervisory Energy Management Strategy for a 12V Vehicle Electrical System
}

\author{
Colin Waldman, Sabarish Gurusubramanian, Lisa Fiorentini, Marcello Canova*
}

\author{
* Center for Automotive Research - The Ohio State University, \\ 930 Kinnear Rd, Columbus OH, 43212, USA.
}

\begin{abstract}
:
This paper describes the development, implementation, and experimental verification of a supervisory energy management strategy for the vehicle electrical system of a passenger car. The control strategy commands the alternator duty cycle such that vehicle fuel economy is optimized whilst the instantaneous load current demand is met and constraints on the system voltage and battery state of charge are satisfied.

The work is based on a control-oriented model of the vehicle electrical system, experimentally validated against vehicle data. Then, a constrained global optimal control problem is formulated for the energy management of the electrical system, and analytically solved using the Pontryagin's Minimum Principle (PMP). The optimal solution obtained is evaluated for a range of different driving cycles and electrical load current profiles, leading to the formulation of an adaptive supervisory control strategy that is implemented and tested in vehicle.
\end{abstract}

Keywords: Automotive, Energy Management, Electrical System, Model-based Control, Optimal Control

\section{INTRODUCTION}

In an effort to comply to the stringent government mandates on fuel economy and $\mathrm{CO}_{2}$ emissions without compromising on vehicle performance and customer acceptability, the automotive industry is striving to rapidly develop and implement a range of design, system integration and control solutions for increasing the energy conversion efficiency of engines and powertrain systems.

The vehicle ancillary loads represent a considerable source of energy dissipation, providing the opportunity to achieve fuel economy benefits at a relatively low cost. Managing the energy consumption of the vehicle loads, such as the air conditioning system, fans and blowers, or the alternator, has in fact been explored recently as a direction towards vehicle fuel economy improvement, see for instance (Silva et al., 2009; Chiara and Canova, 2012; Lyu et al., 2007).

In particular, the vehicle electrical system presents interesting opportunities for energy optimization. In its simplest form, the system consists of a belt driven alternator integrated with a voltage regulator, and a lead-acid battery as the energy storage device. During vehicle operation, the electrical system supplies the electrical power required for the actuation of several vehicle subsystems or components, such as lighting and infotainment, cabin blower, radiator fan, defroster and heated seats.

The alternator is typically controlled to fulfill the demand of the vehicle electrical loads, as well as to recharge

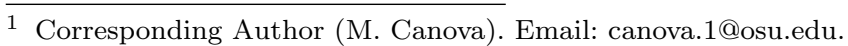

the battery whenever needed. Therefore, the battery is minimally utilized, and limited to starting the engine and to supplement the alternator when high electric load demands occur. However, the energy storage capacity provided by the battery could be exploited for achieving potential fuel savings.

Conceptually, the topology of a vehicle electrical system resembles the architecture of a parallel mild hybrid-electric vehicle, with the exception that the alternator can only absorb power. It is therefore evident that supervisory energy management strategies, such as those developed for the control of hybrid vehicles, could be applied to the vehicle electrical system to improve fuel economy (Shen et al. (2003)). To this extent, an energy-based approach to the control of the vehicle electrical system was proposed initially by Koot et al. (2005); Kessels et al. (2007), where optimization techniques have been applied to design an optimal offline strategy as well as a causal (implementable) strategy. Experimental results obtained on a chassis dynamometer pointed to fuel economy improvement of up to $2.6 \%$ on the New European Driving Cycle (NEDC).

More recently, Couch et al. (2013) proposed a modelbased approach for the energy optimization of a vehicle electrical system, based upon the Equivalent Consumption Minimization Strategy (ECMS), an approach initially developed for the supervisory energy management of charge-sustaining HEVs (Paganelli et al., 2000a,b; Sciarretta et al., 2004; Pisu and Rizzoni, 2007). The simulation results presented show a reduction of engine fuel consumption of up to $1.5 \%$ on the FTP driving cycle. 
This paper describes the development and vehicle implementation of a supervisory energy management strategy for a vehicle electrical system, which controls the alternator duty cycle to minimize the energy consumption while meeting the load current demand from the auxiliary loads. The work is based on a control-oriented model of the $12 \mathrm{~V}$ electrical system of a passenger car, which captures the dynamics of the bus voltage, battery state of charge, and the energy consumed by the alternator.

The supervisory control design is approached by formulating a constrained global optimal control problem, for which an analytical solution is obtained through the Pontryagin's Minimum Principle (PMP) . While the procedure adopted leverages an establish procedure for energy management of hybrid and plug-in hybrid vehicles, see for instance Stockar et al. (2010); Serrao et al. (2011); Stockar et al. (2011); Kim et al. (2011), the application to ancillary loads reduction is novel and presents significant differences in the formulation of input constraints.

Since the PMP theorem provides an optimal solution only with full information on the driving cycle and load current profile, a thorough analysis is conducted to formulate an Adaptive PMP control strategy that can be implemented in real time on a rapid prototyping ECU. Results from vehicle testing are benchmarked against the production control strategy.

\section{MODEL OF THE VEHICLE ELECTRICAL SYSTEM}

The electrical system of a conventional (non hybrid) passenger car typically consists of three main components, namely the battery, the alternator and the electrical loads. As shown in Figure 1, regardless of the specific control strategy implemented, the Vehicle Electrical System (VES) control commands the alternator duty cycle $(D C)$, which in turn generates a field current $\left(I_{f}\right)$ within the alternator. The field current determines the amount of current produced by the alternator $\left(I_{a l t}\right)$, in relation to the engine speed $\left(N_{\text {eng }}\right)$ and the battery voltage $\left(V_{b a t}\right)$. The battery current $\left(I_{b a t}\right)$ is the difference between the load current $\left(I_{\text {loads }}\right)$ and the alternator current. Depending on this current balance, the battery either accepts or supplies current, hence setting the voltage of the system.

Figure 1 presents a block diagram showing the cause and effect relationships of the three interconnected models.

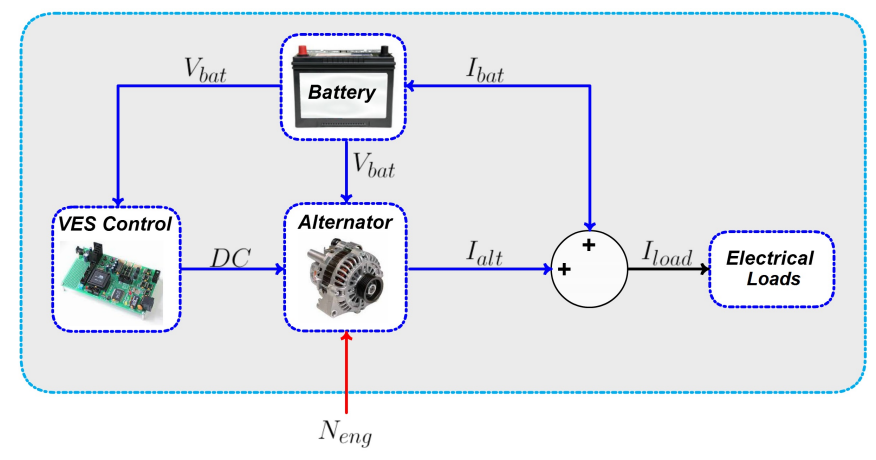

Fig. 1. Schematic of the Vehicle Electrical System.
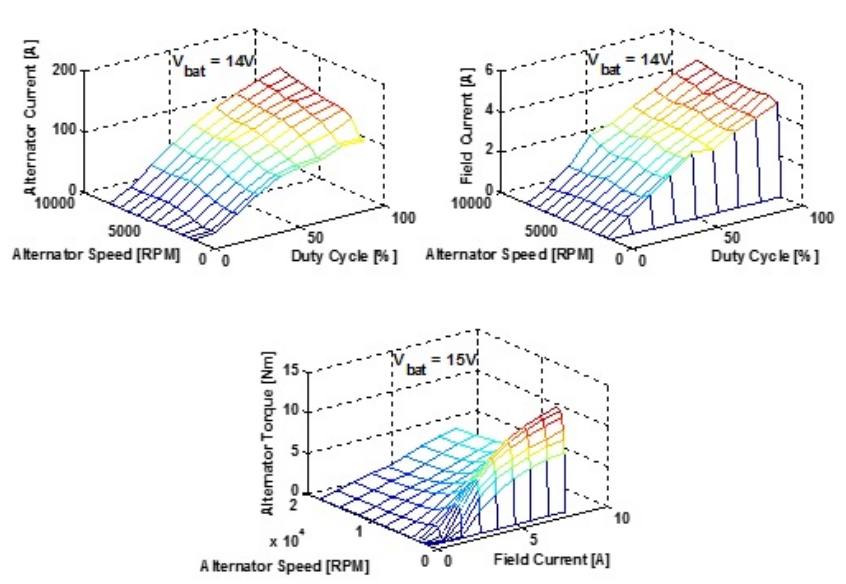

Fig. 2. Alternator Contour Plots for Fixed Battery Voltage.

The work presented in this paper was developed for the electrical system of a passenger car. To this extent, the electrical system of a test vehicle was fully instrumented to allow for real-time measurement and acquisition of the most important operating variables. The models of the alternator and the battery, together with the baseline VES control strategy (Electrical Voltage Regulation, or EVR), will be described in further detail in the following sections.

\subsection{Alternator Model}

A heuristic, map-based model of the alternator was developed for integration into the comprehensive VES model. The alternator model consists of three interconnected lookup tables, which characterize the alternator torque, output current, and field current. The general form of the look-up tables is described in implicit form as:

$$
\begin{gathered}
T_{a l t}=T_{a l t}\left(N_{\text {eng }}, I_{f}, V_{\text {bat }}\right) \\
I_{a l t}=I_{a l t}\left(N_{\text {eng }}, D C, V_{\text {bat }}\right) \\
I_{f}=I_{f}\left(N_{\text {eng }}, D C, V_{\text {bat }}\right)
\end{gathered}
$$

Figure 2 illustrates each output variable for a fixed battery voltage.

The alternator model was identified from experimental data collected on the test vehicle on a chassis dynamometer. The vehicle electrical system was instrumented with shunts to monitor the alternator current, while a programmable load was used to set the battery voltage and the duty cycle was imposed by bypassing the ECU.

\subsection{Battery Model}

The battery is modeled by considering a simple equivalent circuit, as commonly done in applications related to vehicle system energy management, see for instance Kessels et al. (2007); Serrao et al. (2011); Couch et al. (2013). In this study, a standard $12 \mathrm{~V}$ lead-acid battery is considered, and its specifications are summarized in Table 1.

Assuming a first-order equivalent circuit model as a reasonable approximation of the battery low-frequency behavior, the terminal voltage is expressed as: 


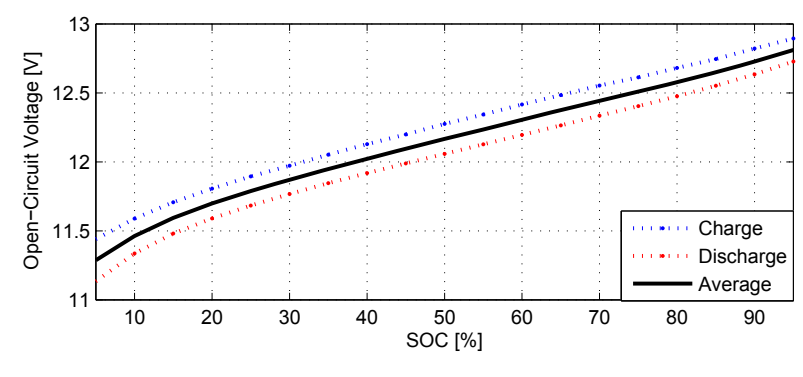

Fig. 3. Battery Open-Circuit Voltage Map for $T_{b a t}=25^{\circ} \mathrm{C}$.

$$
\begin{aligned}
V_{b a t} & =E_{0}-R I_{b a t}-V_{C 0} \\
\frac{d V_{C 0}}{d t} & =-\frac{V_{C 0}}{R_{0} C_{0}}+\frac{I_{b a t}}{C_{0}}
\end{aligned}
$$

where $V_{C 0}$ is the potential difference across the capacitance $C_{0}, E_{0}$ is the open-circuit voltage, $R$ the internal resistance and $R_{0}$ the over-voltage resistance.

The parameters in Equation (4) are functions of several operating variables:

$$
\begin{gathered}
E_{0}=f\left(T_{b a t}, S O C\right) \\
R, R_{0}, C_{0}=f\left(T_{b a t}, S O C, I_{b a t}\right)
\end{gathered}
$$

where $T_{b a t}$ is the battery temperature; and the state of charge (SOC) of the battery is simply computed through current integration:

$$
S O C(t)=S O C_{0}-\frac{1}{A h_{n o m}} \int_{t_{0}}^{t} I_{b a t} d t
$$

where $S O C_{0}$ is the SOC at time $t_{0}$ and $A h_{n o m}$ is the nominal battery capacity.

The parameters shown in Equations 6 and 7 were identified from experimental data. The experimental setup utilized consists of a programmable load and supply system, alongside an environmental chamber to control the temperature throughout the test. Tests were performed at several temperatures, from $-10^{\circ} \mathrm{C}$ to $50^{\circ} \mathrm{C}$ at equal intervals of $5^{\circ} \mathrm{C}$

At each temperature, a set of battery current profiles was engineered to identify the model parameters. Figure 3 shows the identified $E_{0}$ vs SOC curve, while Figure 4 shows an example of the curves representing the parameter $R$ as functions of the SOC and current level at $25^{\circ} \mathrm{C}$.

\subsection{Baseline VES Control}

In order to verify the vehicle electrical system model and provide a baseline for performance and fuel economy comparison, the production control strategy was here modeled. The production strategy (EVR) is a simple voltage regulator, which attempts at maintaining the battery terminal voltage close to a temperature-dependent reference voltage. In particular, the voltage reference value drops as the

\begin{tabular}{|c|c|}
\hline Type & Lead-Acid AGM \\
\hline 20 Hour Capacity & $75 \mathrm{Ah}$ \\
\hline Nominal Voltage & $12 \mathrm{~V}$ \\
\hline Max Charging Current & $120 \mathrm{~A}$ \\
Max Discharging Current & $700 \mathrm{~A}$ \\
\hline
\end{tabular}

Table 1. Summary of Battery Specifications.

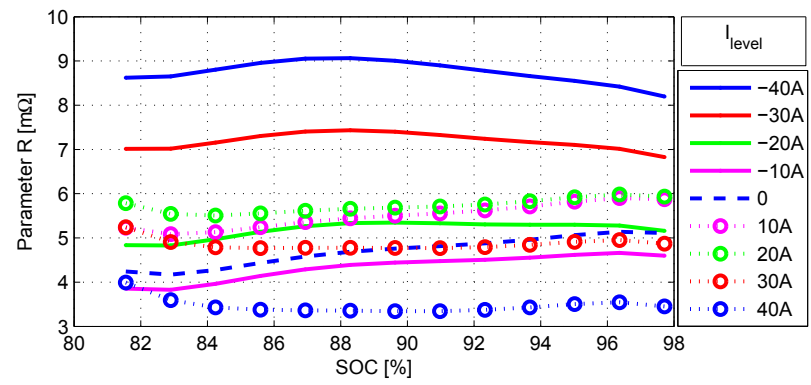

Fig. 4. Parameter $R$ Map for $T_{b a t}=25^{\circ} C$.

battery temperature increases. The above strategy is simply modeled through the implementation and tuning of a gain-scheduled PI controller, with temperature-dependent set-point and gains.

\subsection{Model Validation}

The VES model was verified by comparing the predicted voltage and alternator current to experimental data collected over a range of vehicle operating conditions. Experimental data were collected during highway and city driving conditions, while several electrical loads were activated by the driver to create a realistic load current profile. The vehicle velocity profile, with the corresponding engine speed, torque and vehicle electrical load are shown for in Figure 5.

Figure 6 compares the results of the electrical system model to the experimental battery voltage and alternator current. The model outputs match the test data during the entire driving cycle, and captures well the sudden voltage
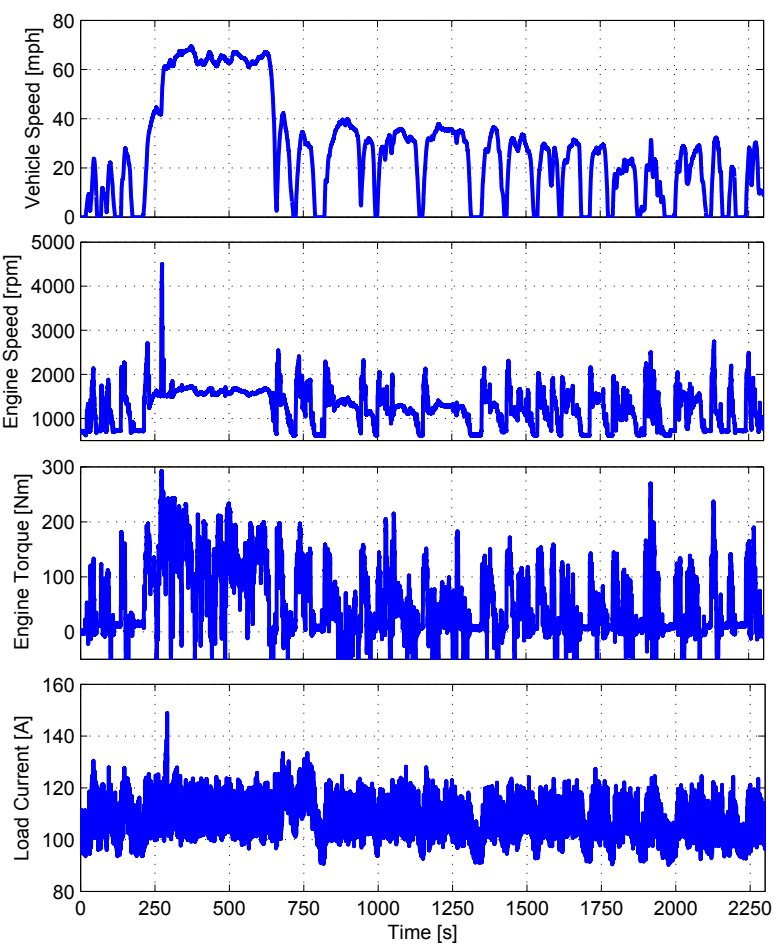

Fig. 5. VES Model Validation: Input Data. 


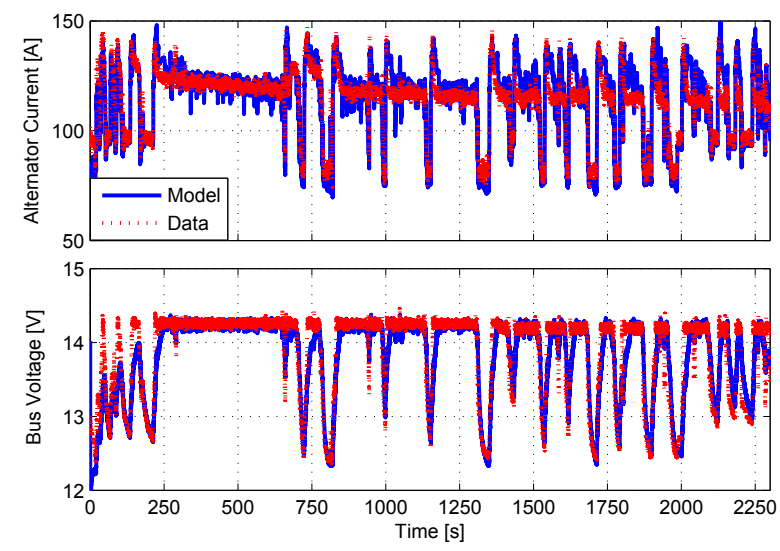

Fig. 6. Vehicle Electrical System Validation: Comparison of Output Data.

drops induced by the rapid fluctuations of the alternator duty cycle caused by the production control strategy.

Overall, the RMS error of the battery voltage is $0.26 \mathrm{~V}$ $(1.8 \%)$, whereas the RMS error of the alternator current is $8 \mathrm{~A}(6 \%)$. Therefore, the vehicle electrical system model can be considered an effective tool for control development and verification.

\section{ENERGY-OPTIMAL CONTROL OF THE VEHICLE ELECTRICAL SYSTEM}

The vehicle electrical system energy management problem is approached as a constrained global optimal control problem over a driving cycle.

\subsection{Problem Definition}

The desired outcome of the energy management strategy under consideration is the minimization of the cumulative vehicle fuel consumption over a given driving cycle, while ensuring that the battery operates nominally in charge sustaining conditions. For this reason, a global optimal control problem is formulated, based upon the following cost function:

$$
J=\int_{t_{0}}^{t_{f}} \dot{m}_{f u e l}(t) d t
$$

where $\dot{m}_{f u e l}(t)$ is the instantaneous fuel consumption of the engine. This term is predicted from the steady-state engine fuel consumption map implemented in the model, as a function of the engine speed and input torque. The effects of the electrical ancillary loads are accounted for by adding the alternator torque to the engine torque, which is a time-varying external input dependent on the specific driving profile. Therefore:

$$
\dot{m}_{\text {fuel }}=L(x(t), u(t), t) \Rightarrow J=\int_{t_{0}}^{t_{f}} L(x, u, t) d t
$$

where $L(\cdot)$ is a continuously differentiable function of the state dynamics of the system, $x(t)$, and the control input, $u(t)$, which is represented by the battery power.

For control purposes, the dynamics of the battery is approximated to a quasi-static system, namely considering only the differential equation that relates the battery SOC to the battery power:

$$
\frac{d}{d t} x(t)=f(x(t), u(t), t)=-\frac{I_{b a t}(t)}{3600 \cdot A h_{n o m}}
$$

The state of charge dynamics may be reformulated as a function of both the control command and system state utilizing the simplified battery model discussed above:

$$
\begin{gathered}
V_{b a t}=E_{0}-R_{e} I_{b a t} \\
\phi(t)=I_{b a t}(t)=\frac{E_{0}-\sqrt{E_{0}^{2}-4 R_{e} P_{b a t}(t)}}{2 R_{e}} \\
\frac{d}{d t} x(t)=f(x(t), u(t), t)=-\frac{\phi(x, u, t)}{3600 \cdot A h_{\text {nom }}}
\end{gathered}
$$

where $R_{e}=R+R_{0}$ and the parameters are functions of temperature, SOC and current level, computed at each time step.

In order to avoid overcharging or overdischarging of the battery, a set of constraints are implemented on the state of charge:

$$
\begin{aligned}
& x\left(t_{0}\right)=x_{0} \\
& x\left(t_{f}\right)=x_{f}=x_{0} \\
& x_{\text {min }} \leq x(t) \leq x_{\text {max }}
\end{aligned}
$$

Furthermore, constraints on the inputs must be accounted for, e.g., to limit the minimum and maximum battery and alternator current.

In addition, a specific set of constraints must be included in the problem to maintain driver comfort and safety, and to avoid malfunctioning of the coupled electrical loads:

(1) The load current demand from the electrical loads must be satisfied at all times:

$$
I_{\text {load }}(t)=I_{\text {bat }}(t)+I_{\text {alt }}(t)
$$

(2) The battery voltage must be monitored to avoid overcharging and overdischarging the battery and to ensure that resistive loads are properly powered:

$$
V_{\text {min }} \leq V_{\text {bat }}(t) \leq V_{\max }(t)
$$

(3) The rate of change of the battery voltage must be limited to prevent flickering or other malfunctioning caused by rapid voltage fluctuations. This constraint can be mathematically formulated as:

$$
\left|\frac{d V_{b a t}}{d t}\right| \leq V_{r}^{*}
$$

In this work, $x_{\min }=80, x_{\max }=90, V_{\min }=11 \mathrm{~V}$, $V_{\max }=14.6 \mathrm{~V}$, and $V_{r}^{*}=0.1 \mathrm{~V} / \mathrm{s}$.

The implementation of the input constraints is carried out by defining a current-split factor $\gamma$, which represents all possible current-split options for the vehicle electrical system:

$$
\begin{aligned}
\gamma & =[0,1] \in \mathrm{R}^{n} \\
I_{\text {alt }}(t) & =\gamma \cdot I_{\text {alt }, \text { max }} \\
I_{\text {bat }}(t) & =I_{\text {loads }}(t)-I_{\text {alt }}(t)
\end{aligned}
$$

where $I_{a l t, \max }$ is the maximum current (i.e. the corresponding to a $100 \%$ duty cycle), that the alternator can produce at the considered operating condition $\left(N_{\text {eng }}, V_{\text {bat }}\right)$. The resulting battery current array is then filtered to eliminate all the current split options that violate 


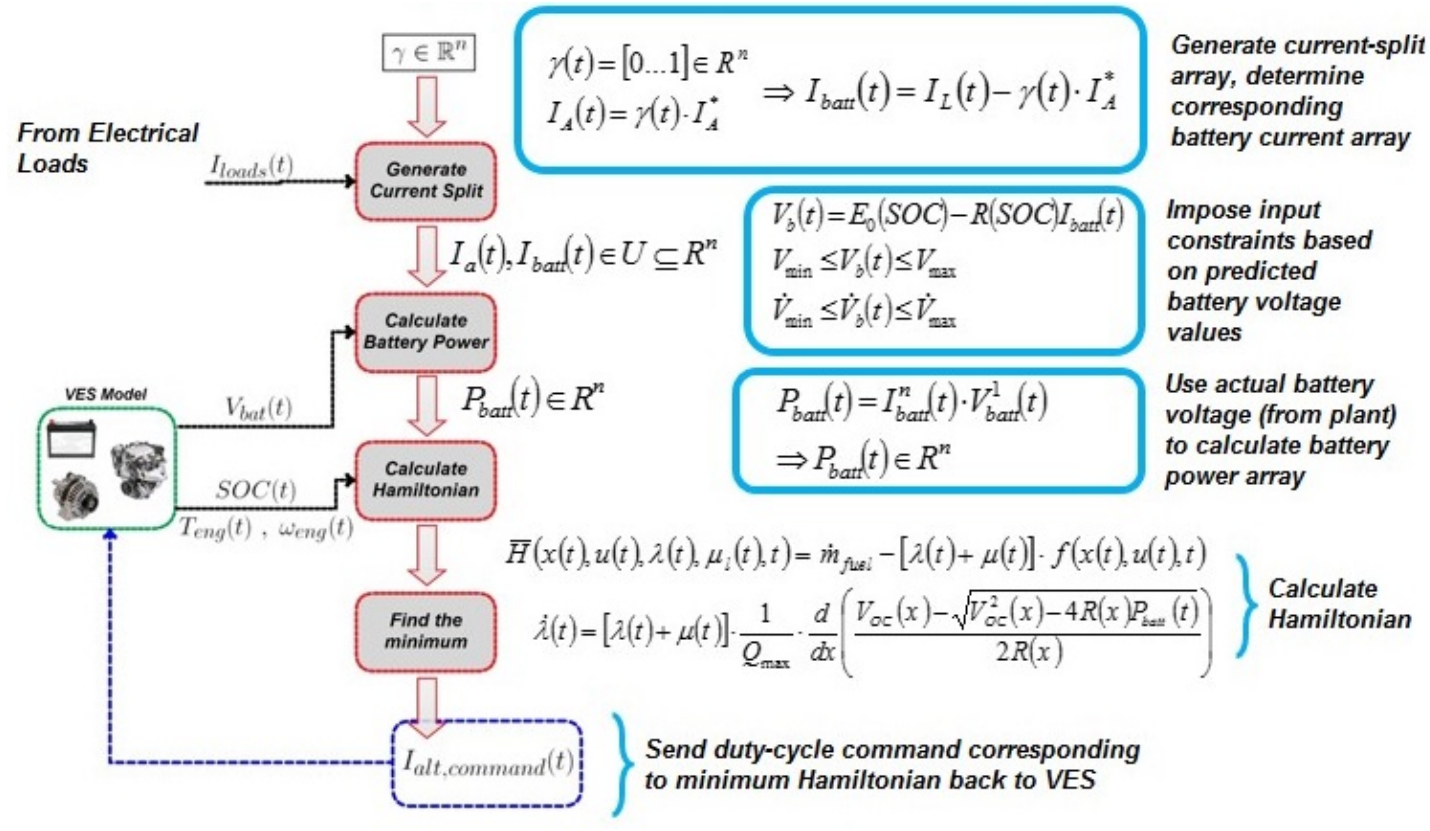

Fig. 7. Structure of PMP Control Strategy.

the above constraints. Using (12), the voltage constraints can be expressed as:

$$
\begin{aligned}
& V_{\text {bat }}(t)=E_{0}-R_{e} I_{b a t}(t) \in\left[V_{\text {min }}, V_{\text {max }}\right] \\
& \left|V_{\text {bat }}(t)-V_{\text {bat }}(t-\delta t)\right| \leq V_{r}^{*} \cdot \Delta t
\end{aligned}
$$

where $\Delta t=1 s$

\subsection{Solution Method: Pontryagin's Minimum Principle}

The constrained global optimal control problem described above is here solved by applying the Pontryagin's Minimum Principle (PMP) Geering (2007).

The theorem allows one to approximate the problem as a local optimization problem by introducing a set of necessary conditions that may be cast as follows (Geering, 2007):

$$
\begin{aligned}
& H\left(x^{*}, u^{*}, \lambda^{*}, \mu^{*}, t\right) \leq H(x, u, \lambda, \mu, t) ; \forall u \neq u^{*} \\
& x^{*}\left(t_{f}\right)=x^{*}\left(t_{0}\right)=x_{0} \\
& \dot{x}^{*}(t)=\left.\nabla_{\lambda} H\right|^{*} \\
& \dot{\lambda}^{*}(t)=-\left.\nabla_{x} H\right|^{*}
\end{aligned}
$$

where $H(x, u, \lambda, \mu, t)$ denotes the extended Hamiltonian function, which is described as:

$$
H(x, u, \lambda, \mu, t)=\dot{m}_{f u e l}-[\lambda(t)+\mu(t)] \cdot \frac{\phi(x, u, t)}{3600 \cdot A h_{n o m}}
$$

In Equation (18), $\lambda(t)$ is the Lagrange multiplier describing the costate dynamics of the problem, and $\mu$ is introduced to account for the state constraints on the battery SOC :

$$
\begin{gathered}
\dot{\lambda}(t)=-\nabla_{x} \bar{H} \\
\mu(t)= \begin{cases}\mu_{l}: & S O C \geq S O C_{\max } \\
-\mu_{l}: & S O C \leq S O C_{\min } \\
0 \quad: \quad \text { else }\end{cases}
\end{gathered}
$$

The penalty term $\mu_{l}$ is a constant value that must be calibrated to prevent the control strategy from allowing the battery SOC to exit the upper or lower bound.
It is well known that, while the PMP provides only a set of necessary conditions for optimality, a very good approximation of the optimal solution is obtained if a value for the initial condition $\lambda_{0}=\lambda(t=0)$ that satisfies the above conditions is found (Serrao et al., 2011). This can be done offline (hence with full knowledge of the vehicle driving profile and of the load current profile), using a single shooting method. In the shooting method, the initial condition $\lambda_{0}^{*}$ that satisfies the necessary conditions of the PMP is obtained simply by "sweeping" across a range of values of $\lambda$ to identify the value for which $x\left(t_{f}\right)=x_{0}$.

The solution based on the PMP theorem is implemented in discrete form, assuming a sample time $\Delta t=1 \mathrm{~s}$. A flow chart showing the control implementation is shown in Figure 7.

\section{RESULTS AND ANALYSIS OF PMP SOLUTIONS}

In order to gather a comprehensive understanding of the general behavior of the vehicle electrical system and evaluating the potential for fuel economy improvement, the solution developed using the PMP approach is studied indepth for a range of different driving cycles and electrical load current profiles.

\subsection{Analysis of Solution for the ECE15 Case Study}

As a first step, the ECE15 regulatory driving cycle with a constant electrical load demand of $57.5 \mathrm{~A}$ is considered.

This driving cycle was selected due to the simplicity of its velocity profile, which assists in illustrating the behavioral characteristics of the optimal control solution, whereas a constant electrical load of $57.5 \mathrm{~A}$ was chosen to decouple the effects of the state dynamics from that of the instantaneous fuel consumption, while approximating the average value of the experimental electrical load measured during realistic driving conditions. The vehicle velocity 

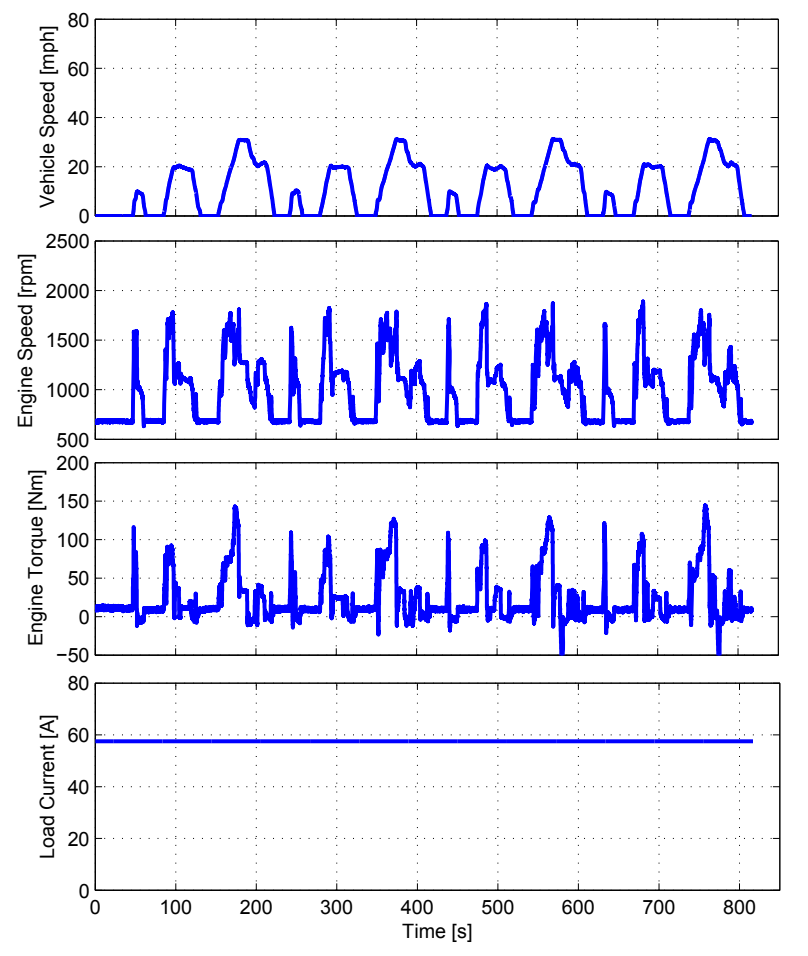

Fig. 8. Summary of Input Profiles for the ECE15 Scenario with Constant Load Current.

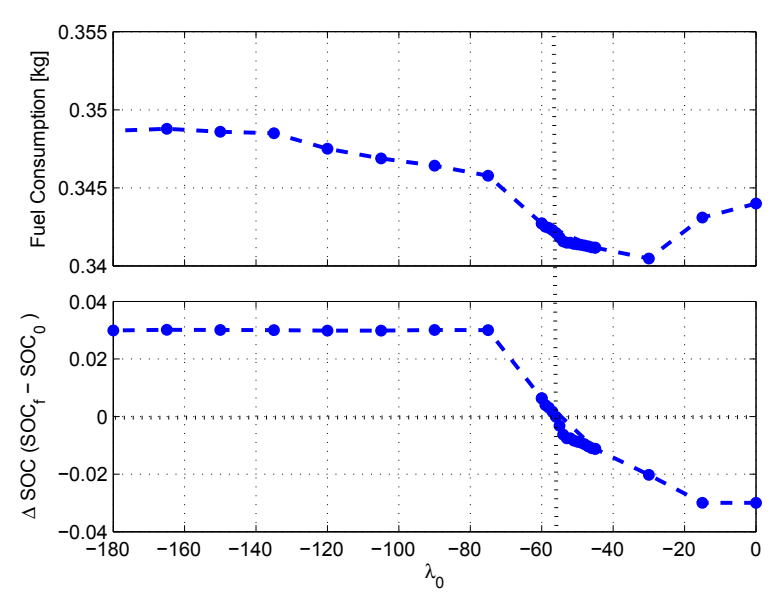

Fig. 9. Result of Shooting Method for NEDC with Constant Load Current of 57.5A.

profile, engine speed and torque traces, and the electrical load may be observed in Figure 8 .

The initial condition $\lambda_{0}^{*}$ that satisfies the necessary conditions of the PMP is obtained using the single shooting method. As shown in Figure 9, the value $\lambda_{0}^{*}=-56$ allows for the aforementioned scenario to satisfy all the necessary conditions of the PMP theorem, including the constraint of the battery SOC given by Eq.(15).

The behavior of the vehicle electrical system resulting from this solution is summarized in Figure 10. For this scenario, it can be observed from Figure 10(a) that the co-state $\lambda(t)$ is essentially constant through the driving profile, varying

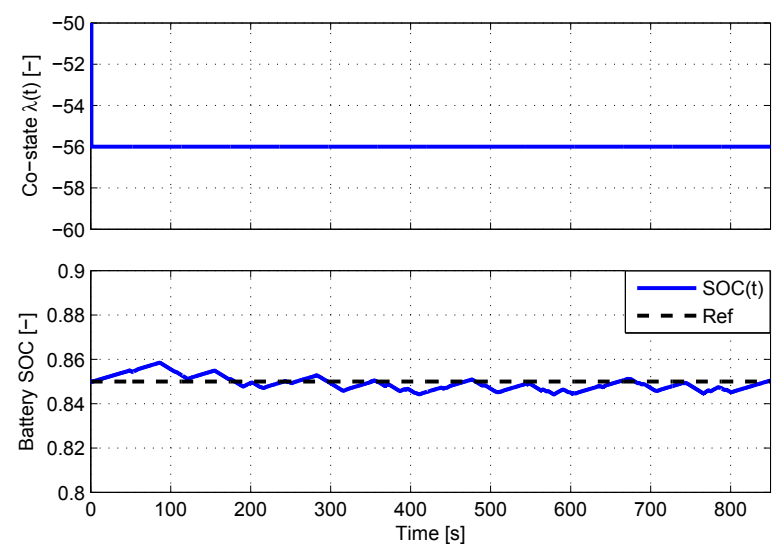

(a) Lagrange Multiplier and Battery SOC
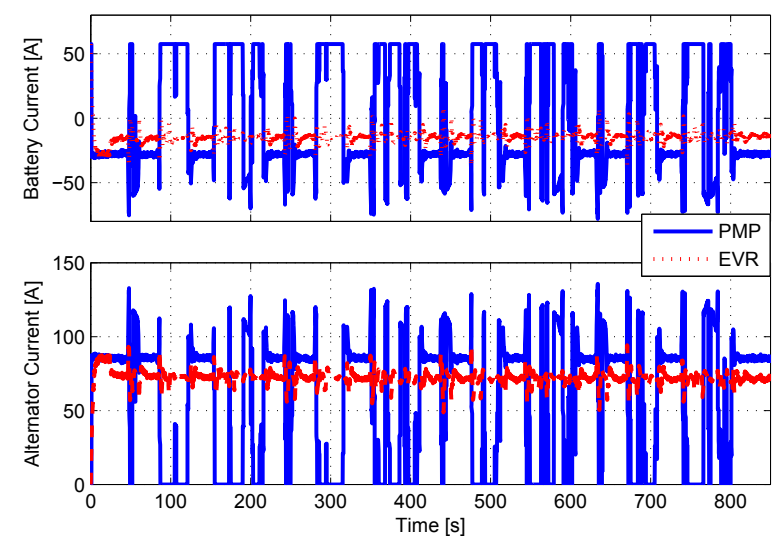

(b) Battery and Alternator Current

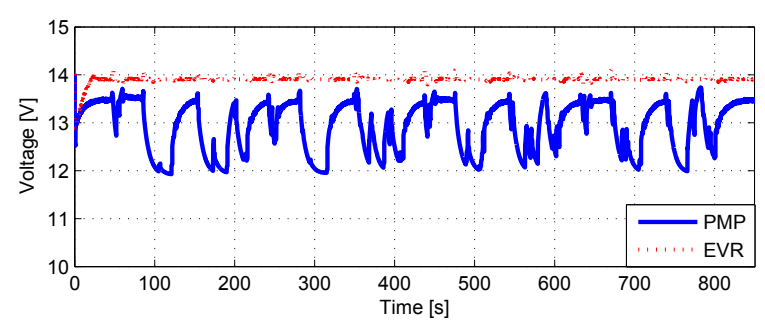

(c) Bus Voltage

Fig. 10. Comparison of Optimal Solution and Baseline EVR Strategy for ECE15 Cycle with Constant 57.5A Load Current Scenario.

by less than $0.01 \%$. This trend is not limited to this case, but rather it was systematically found for several driving and load current scenarios.

The charge-sustaining behavior of the battery may be also observed in Figure 10(a), in particular it can be verified that the optimal control limits the fluctuations of the SOC and enforces the final state constraint.

The trajectory of the Lagrange multiplier and of the battery SOC reveal important information on the behavior of the VES when the optimal control policy is applied. Specifically, the co-state dynamics can be neglected, hence simplifying considerably the optimal control problem to a static optimization at each time step, ultimately providing 


\begin{tabular}{|c|c|}
\hline Controller & Fuel Economy \\
\hline EVR & $19.1 \mathrm{mpg}$ \\
\hline PMP & $19.5 \mathrm{mpg}$ \\
\hline Improvement & $2.1 \%$ \\
\hline
\end{tabular}

Table 2. Summary of Fuel Economy for EVR and PMP Solution (ECE15 Cycle, Constant Load Current).

an opportunity for on-line implementation through the design of adaptive control schemes.

The observed behavior results from the relatively small utilization of the battery energy in relation with the nominal capacity, and is confirmed by the SOC trajectory. To some extent, this conclusion is also supported by similar results obtained for the case of optimal control for chargesustaining hybrid electric vehicles, where the battery pack capacity is only minimally utilized (Serrao et al., 2009, 2011).

Figure 10(b) shows the battery and alternator current profiles prescribed by the PMP solution for this case, and compares the results to the benchmark EVR control strategy. As expected, the optimal solution leads to a significantly increased use of the battery, together with a "bang-bang" behavior.

This phenomenon can be explained by jointly looking at Figure 10(b) and Figure 8 and is rather intuitive: the control policy generally attempts at using the battery instead of the alternator to provide energy to the electrical load at higher fuel inection rates, while taking advantage of the deceleration events, when energy from the alternator is cheaper, to recharge the battery as much as possible. In any case, the current draw from the battery never exceeds $50 \mathrm{~A}$, which is a very small compared to the maximum discharging current indicated in Table 1.

Finally, Figure 10(c) compares the battery voltage for the EVR and the optimal policy. As expected, the PMP solution forces the bus voltage to fluctuate significantly during the test, as opposed to the EVR control, even though the constraints on the voltage and voltage rate of change are enforced at all times.

In summary, the solution obtained from the PMP theorem leads to a significant increase of the battery usage, which leads to a $2.1 \%$ improvement in fuel economy, compared to the baseline production control strategy. Table 2 provides the details of the overall fuel economy benefits realized with the optimal control solution.

\subsection{Sensitivity Analysis of Optimal Solution}

To understand how the VES operates under energyoptimal conditions, the analysis described above for the ECE15 cycle was expanded to encompass numerous combinations of driving profiles and electrical loads.

In particular, several driving profiles have been considered, while the electrical load was set to constant values. For each driving cycle/electrical load scenario, the optimal solution was computed with the shooting method, and the results are summarized in Table 3 and Table 4 , reporting the improvement in fuel economy over the baseline control strategy.

\begin{tabular}{|c|c|c|c|c|c|}
\hline Driving Cycle $\quad$ Load (A) & 30 & 40 & 57.5 & 75 & 90 \\
\hline FTP & -59 & -61 & -61 & -80 & -105 \\
\hline NEDC & -27 & -28 & -56 & -70 & -90 \\
\hline Artemis & -54 & -57 & -58 & -77 & -97 \\
\hline Indian Urban & -20 & -57 & -66 & -76 & -91 \\
\hline $\mathrm{JC08}$ & -49 & -53 & -68 & -78 & -100 \\
\hline $\begin{array}{l}\text { US06 } \\
\end{array}$ & -60 & -76 & \begin{tabular}{|l|}
-89 \\
\end{tabular} & -105 & -112 \\
\hline
\end{tabular}

Table 3. Optimal Co-State Initial Condition $\lambda_{0}^{*}$ for Different Scenarios.

\begin{tabular}{|c|c|c|c|c|c|}
\hline Load (A) & 30 & 40 & 57.5 & 75 & 90 \\
\hline Driving Cycle & 2.0 & 2.0 & 1.8 & 1.7 & 1.7 \\
\hline FTP & 1.8 & 1.7 & 2.1 & 2.2 & 2.2 \\
\hline NEDC & 1.2 & 1.2 & 1.6 & 1.7 & 1.7 \\
\hline Artemis & 1.2 & 1.7 & 1.8 & 1.9 & 1.9 \\
\hline Indian Urban & 1.8 & 1.9 & 1.8 & 1.9 & 1.7 \\
\hline JC08 & 0.6 & 1.0 & 1.1 & 1.0 & 1.0 \\
\hline US06
\end{tabular}

Table 4. Fuel Economy Improvement over

Baseline Control (EVR) For Different Scenarios (All Values in \%).

Based on the simulation results summarized in the tables, the PMP solution, consistently provides fuel economy improvements up to $2.2 \%$.

Of particular interest is the relation between the initial co-state value $\lambda_{0}^{*}$, the driving cycle, and the electrical load demand. At lower electrical load demands, there is a slight variability in the parameter $\lambda_{0}^{*}$ in relation with the vehicle velocity profile, particularly for the NEDC and Indian Urban driving cycles.

However, as the electrical load demand is increased, the dependence of $\lambda_{0}^{*}$ from the driving condition becomes negligible, leaving the electrical load demand as the primary factor influencing the optimal initial co-state value.

To this extent, it is worth noting that normal vehicle operations result into approximately 40-50A drawn constantly from the VES, without the use of the HVAC system. The radiator fan and cabin blower both require an additional current of 30-40A. Since the primary objective of the control strategy is to meet the electrical load demand at all times, it can be easily observed that, as the current demand increases, it becomes the predominant factor affecting the optimal control solution.

Numerous analyses were performed in simulation to evaluate the sensitivity of the solution to different factors, for instance the initial condition $\lambda_{0}$, the initial SOC, and the SOC bounds. For brevity, the details of this study have been omitted but the main conclusions are summarized as follows:

(1) The parameter $\lambda_{0}^{*}$ presents an inverse linear relationship with the electrical load demand, and very limited dependence on the driving conditions (such as vehicle speed, engine speed/torque profiles;)

(2) The charge-sustaining behavior of the control strategy presents limited sensitivity to the initial condition $\lambda_{0}$

(3) The co-state dynamics $\lambda(t)$ can be reasonably approximated as constant for each of the tested scenarios, which allows for simplifying the optimal control prob- 


\begin{tabular}{|c|c|}
\hline$I_{\text {load }}(A)$ & $\lambda_{0}$ \\
\hline 30 & -55.6 \\
\hline 40 & -61.9 \\
\hline 57.5 & -70.8 \\
\hline 75 & -86 \\
\hline 90 & -104.4 \\
\hline
\end{tabular}

Table 5. Lookup Table for Parameter $\lambda_{0}^{*}$

lem to a static optimization problem at each time step.

The above considerations can be leveraged to develop a "forward-looking" control strategy that can be implemented on a vehicle without prior knowledge of the load current or velocity profile.

\section{DESIGN OF ADAPTIVE PMP CONTROL STRATEGY}

In order to develop a VES control strategy for optimal fuel economy, the implementation of the PMP theorem presented earlier is modified into an adaptive control.

The first step is to neglect the co-state dynamics, leading to the following approximation of the extended Hamiltonian function:

$H \simeq \dot{m}_{\text {fuel }}-\left(\hat{\lambda}+\mu_{l}\right) \cdot \frac{1}{3600 A h_{n o m}} \cdot \frac{E_{0}-\sqrt{E_{0}^{2}-4 R_{e} P_{b a t}}}{2 R_{e}}$

where the parameter $\hat{\lambda}$ is adaptively tuned to ensure charge-sustaining behavior and improvement of vehicle fuel economy.

Leveraging the results of the analysis conducted above, the parameter $\hat{\lambda}_{0}$ is expressed as follows:

$$
\hat{\lambda}=K_{S O C} \cdot\left(S O C_{r e f}-S O C_{T_{s 1}}\right)+\lambda_{0}^{*}\left(T_{s 2}\right)
$$

where $T_{s 1}$ and $T_{s 2}$ denotes that the adaptation based upon the battery SOC and load current occurs at different sample times.

The parameter $\lambda_{0}^{*}$ is a function of the electrical load demand. For implementation, the load current measured on the vehicle is low-pass filtered and passed through a look-up table to determine the corresponding value of the parameter $\lambda_{0}^{*}$. The look-up table is summarized in Table 5.

The adaptation based on the load current is updated at a discrete sampling time $T_{s 2}=T_{s}\left(I_{\text {load }}\right)$, which can be calibrated in simulation to approximate the optimal solution. While this provides for an educated projection of the charge-sustaining control solution, the resulting control strategy can not guarantee that the battery state of charge will depart from the reference value $S O C_{r e f}$.

To this extent, an additional correction is applied, based on a SOC feedback Onori et al. (2010). The correction is represented by a proportional term in Equation (23), where the gain $K_{S O C}$ is a tunable parameter.

The SOC-based adaptation is updated at a sampling time $\left(T_{s 1}=T_{s}(\Delta S O C)\right)$ and tuned to allow the vehicle to make use of the available energy buffer without causing a drift in the battery SOC.

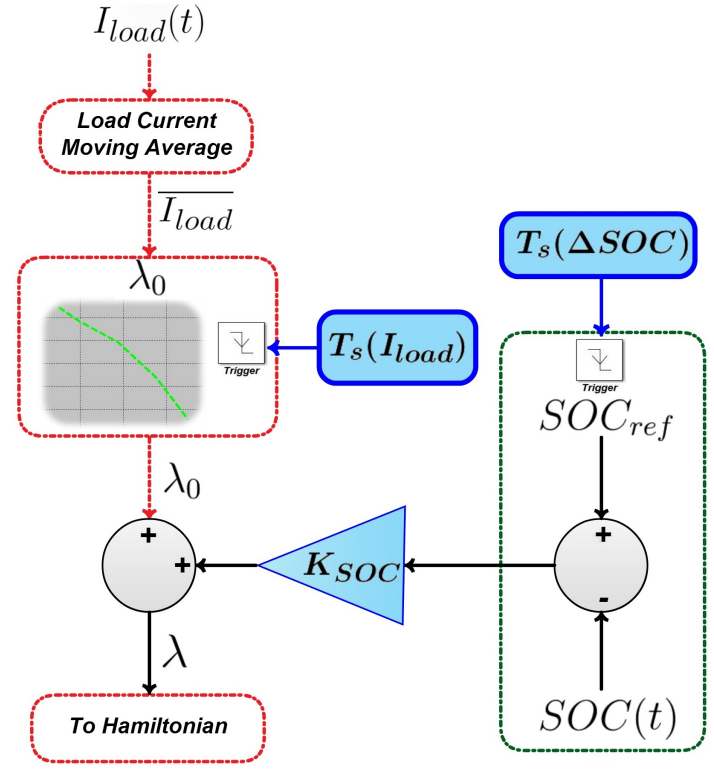

Fig. 11. Schematic of the A-PMP Control Structure.

\begin{tabular}{|l|c|c|}
\hline Description & Parameter & Value \\
\hline Sampling Time & $T_{s 1}$ & $120 \mathrm{~s}$ \\
\hline Sampling Time & $T_{s 2}$ & $15 \mathrm{~s}$ \\
\hline SOC Gain & $K_{S O C}$ & 250 \\
\hline Reference SOC & $S O C_{r e f}$ & $85 \%$ \\
\hline Minimum SOC & $S O C_{\min }$ & $80 \%$ \\
\hline Maximum SOC & $S O C_{\max }$ & $90 \%$ \\
\hline
\end{tabular}

Table 6. Summary of A-PMP Control Strategy Parameters.

Calibration of the SOC gain and update times for the SOC correction and the $\lambda_{0}$ lookup table is necessary to balance fuel economy benefit and battery performance. For example, if too high a value is selected for $K_{S O C}$ the battery SOC dynamics will be restricted and fuel economy benefits may be significantly reduced, however if too low a value is chosen the battery state of charge may drift to either the upper or lower threshold, which may again reduce fuel economy. Figure 11 illustrates the structure of the Adaptive PMP (A-PMP) control strategy, while Table 6 summarizes the parameters selected for implementation.

\section{EXPERIMENTAL TESTING AND RESULTS OF A-PMP CONTROL STRATEGY}

The Adaptive-PMP control strategy was implemented and experimentally verified on the test vehicle. The vehicle was installed on a chassis dynamometer, and an ETAS rapid prototyping and data acquisition platform was adopted to implement and verify alternator control algorithms. Specifically, as illustrated in Figure 12, an ETAS ES1000.3 rapid prototyping platform coupled with Intecrio software was used for data acquisition and control testing. Shunts routed to an ES1303 A/D board were used for measurement of the battery current, alternator current, and electrical load demand, while an ETK interface was employed to gain direct access to the control variables and parameters of the vehicle ECU. 


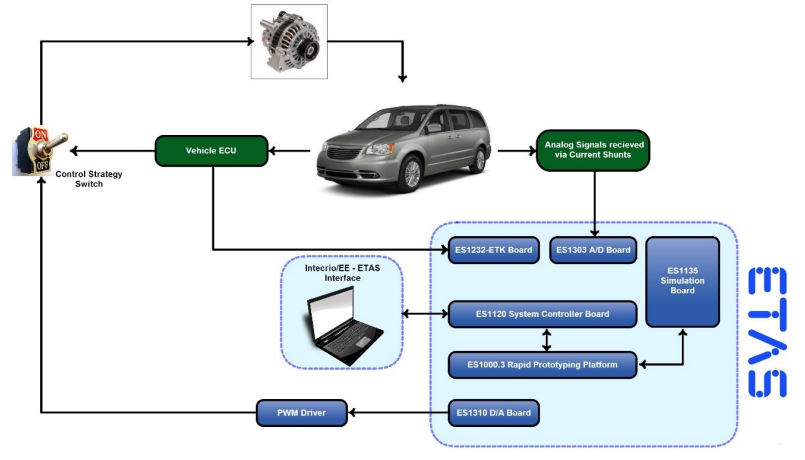

Fig. 12. Schematic of the Experimental Setup.

In addition, an ETAS ES1310 D/A board in conjunction with a PWM driver was adopted to override the production control strategy for the vehicle alternator. This feature allows one to implement and test control algorithms for the electrical system. Finally, A Bosch Intelligent Battery Sensor (IBS) was installed on the $12 \mathrm{~V}$ battery. The IBS is based upon an adaptive, linear Kalman filter estimator, and outputs the battery state of charge with a resolution of $\pm 1 \%$.

The test vehicle was installed on a light-duty chassis dynamometer to calibrate and verify the A-PMP control strategy over numerous regulatory and random errand driving cycles. The vehicle test data were also used as inputs to the baseline VES model (with the EVR production control strategy), to benchmark the performance.

Two different experiments are here shown, namely an EPA fuel economy driving schedule and an errand drive cycle. The EPA driving cycle was performed to the standard testing procedures, namely with all the electrical accessory loads turned off. The latter cycle emulates a real-world driving scenario where both the vehicle speed and the electrical load demand are randomly varied.

Figure 13 summarizes the results for the EPA cycle. The load current profile for this test includes only the electrical loads essential for the engine and vehicle operation. The visible fluctuations of the current demand are caused by the cycling of the radiator fan.

A closer inspection of the alternator and battery current profiles in Figure 13(b) indicates that the adaptive control strategy still produces an overall "bang-bang" response, although with reduced chattering compared to the earlier simulation results.

The "bang-bang" response results from the control strategy attempting at maximizing the use of the alternator when the fuel consumption penalty is limited or zero (for instance, during deceleration and braking events where fuel cut off), while relying on the battery to provide power to the auxiliary loads for the remaining conditions.

The increased utilization of the battery causes significant voltage fluctuations, as shown in Figure 13(c). Here, the switching behavior of the control strategy is reflected by the rapid variations in the voltage, despite the input constraints imposed.

The battery SOC slowly fluctuates during the test, while remaining nominally charge-sustaining (within the accu-
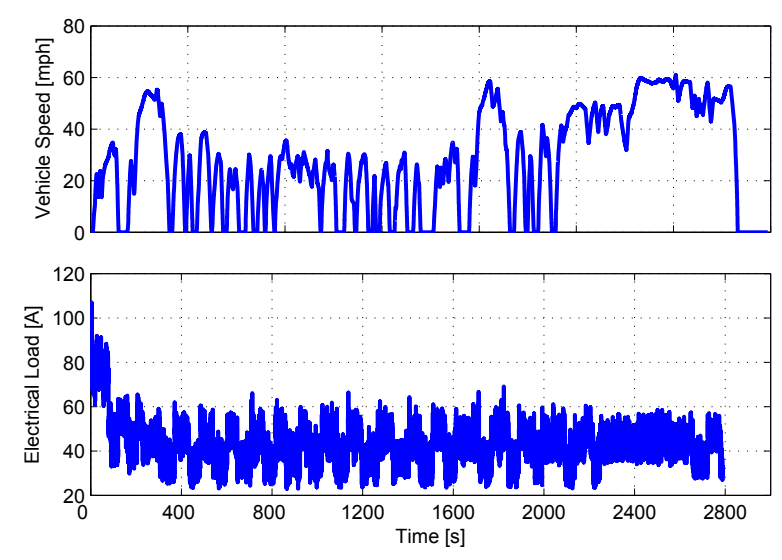

(a) Vehicle Velocity and Electrical Load Profile
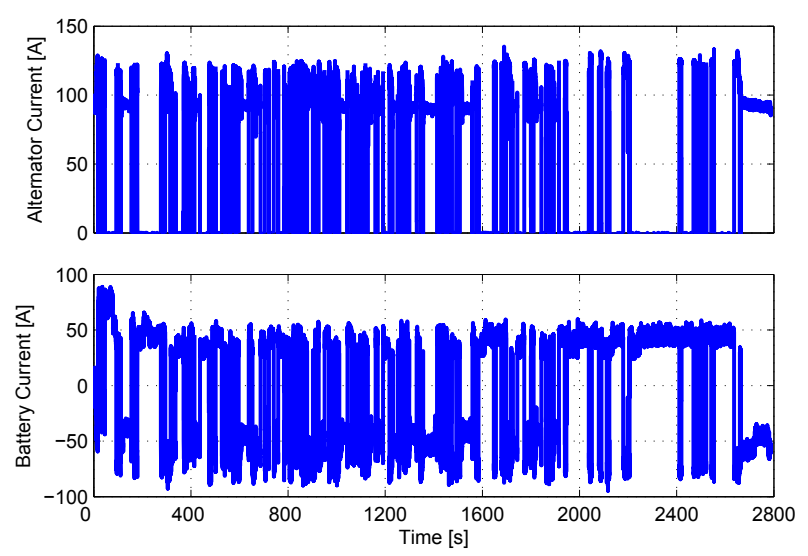

(b) Alternator and Battery Current
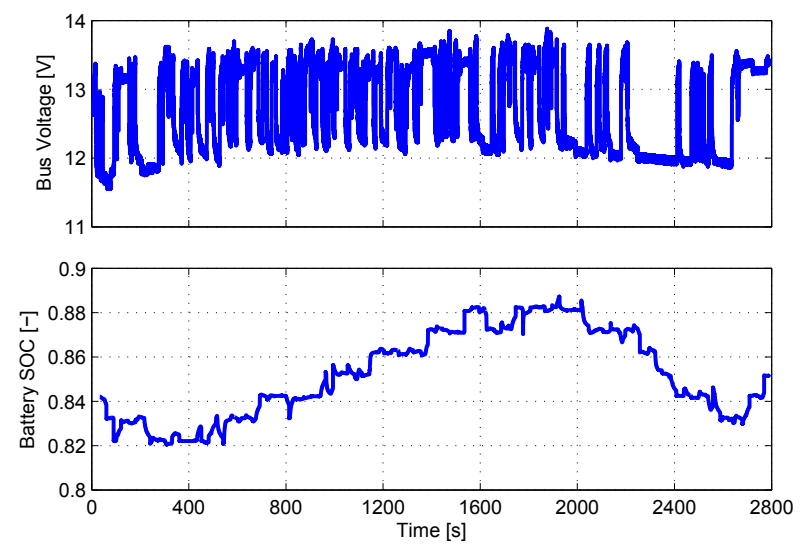

(c) Bus Voltage and Battery SOC

Fig. 13. Experimental Results for A-PMP Control Strategy: EPA Regulatory Driving Cycle.

racy of the SOC estimator). In particular, the control strategy tends to charge the battery during the city portion of the EPA cycle by taking advantage of the frequent stopping events, while it slowly discharges during the highway portion to improve the fuel economy.

Similar results are obtained for the test summarized in Figure 14, based upon a velocity profile that includes urban and extra-urban driving segments. As shown in 

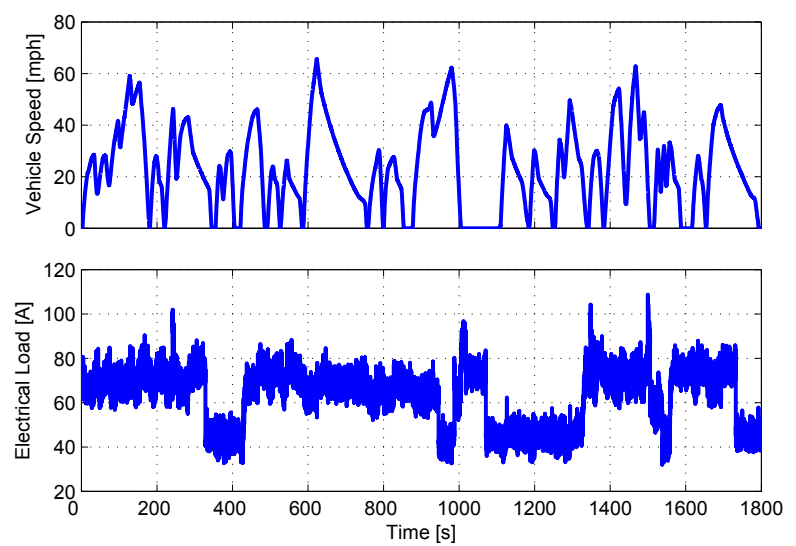

(a) Vehicle Velocity and Electrical Load Profile
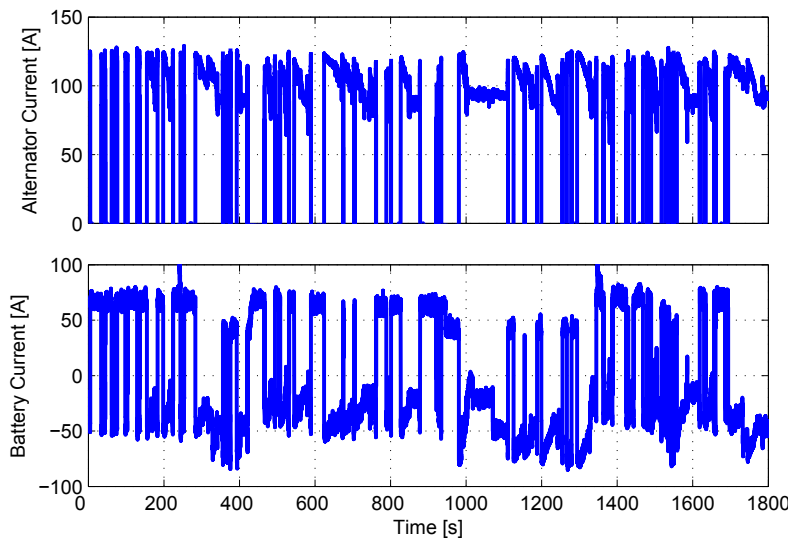

(b) Alternator and Battery Current
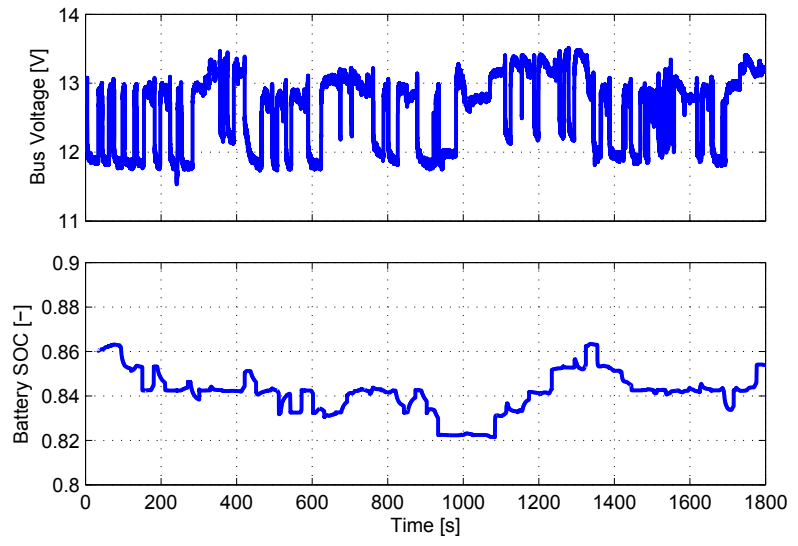

(c) Bus Voltage and Battery SOC

Fig. 14. Experimental Results for A-PMP Control Strategy: Errands Driving Profile.

Figure 14(a), the load current profile varies significantly during the test, representative of a more realistic scenario.

The control strategy produces very similar results, with increased usage of the battery to offset the operation of the alternator. Similarly to the previous case, the A-PMP controller maintains the bus voltage between $12 \mathrm{~V}$ and $13.5 \mathrm{~V}$, which an acceptable range for proper operation of the vehicle electrical system, as well as for ensuring

\begin{tabular}{|l|l|l|l|}
\hline \multicolumn{3}{|c|}{ Length of Driving Cycle } & $21.3 \mathrm{mi}$ \\
\hline \multicolumn{3}{|c|}{ Baseline Strategy Fuel Consumption } & $2221 \mathrm{~g}$ \\
\hline \multicolumn{3}{|c|}{ Baseline Strategy Fuel Economy } & $26.4 \mathrm{mpg}$ \\
\hline \multicolumn{2}{|c|}{ Fuel Economy Improvement (\%) } & 1.0 \\
\hline $\begin{array}{l}\text { Alternator Energy Con- } \\
\text { sumption (MJ) }\end{array}$ & 2.27 & 1.29 & 1.54 \\
\hline $\begin{array}{l}\text { Alternator Fuel Con- } \\
\text { sumption (g) }\end{array}$ & 51 & 24 & 28 \\
\hline $\begin{array}{l}\text { Net Fuel Economy } \\
\text { (mpg) }\end{array}$ & 25.8 & 26.1 & 26.0 \\
\hline $\begin{array}{l}\text { Energy moved through } \\
\text { Battery (kWh) }\end{array}$ & 0.14 & 0.47 & 0.49 \\
\hline
\end{tabular}

Table 7. Summary of Fuel Economy for the EPA Driving Profile.

\begin{tabular}{|l|l|l|l|}
\hline \multicolumn{3}{|c|}{ Length of Driving Cycle } & $11.7 \mathrm{mi}$ \\
\hline \multicolumn{3}{|c|}{ Baseline Strategy Fuel Consumption } & $1440 \mathrm{~g}$ \\
\hline \multicolumn{3}{|c|}{ Baseline Strategy Fuel Economy } & $22.3 \mathrm{mpg}$ \\
\hline \multicolumn{3}{|c|}{ Fuel Economy Improvement (\%) } & 1.4 \\
\hline Control Strategy & EVR & PMP & A-PMP \\
\hline $\begin{array}{l}\text { Alternator Energy Con- } \\
\text { sumption (MJ) }\end{array}$ & 1.74 & 0.99 & 0.99 \\
\hline $\begin{array}{l}\text { Alternator Fuel Con- } \\
\text { sumption (g) }\end{array}$ & 42 & 22 & 22 \\
\hline $\begin{array}{l}\text { Net Fuel Economy } \\
\text { (mpg) }\end{array}$ & 21.7 & 22 & 22 \\
\hline $\begin{array}{l}\text { Energy moved through } \\
\text { Battery (kWh) }\end{array}$ & 0.12 & 0.3 & 0.3 \\
\hline \multicolumn{1}{|c|}{ (kable 8. Summary } & & \\
\hline
\end{tabular}

Table 8. Summary of Fuel Economy for the "Errands" Driving Profile.

that the engine will start after a vehicle shut down. The battery SOC is again nominally charge-sustaining, although fluctuating within a small range around the reference condition.

Finally, Tables 7 and 8 summarize the fuel economy improvements achieved by the A-PMP control strategy for the two test cases considered, and provides a comparison against the baseline EVR controller and to the optimal solution for each case.

The fuel economy was calculated by simulating each driving cycle without the electrical system to determine a baseline. The fuel consumption for the EVR and PMP case were determined in simulation, using the engine speed, torque and load current profiles generated from the experimental tests. In addition to with the fuel consumption metrics, the tables report the overall energy moved through the battery, to illustrate the differences in its utilization.

In both cases, the A-PMP control strategy leads to a fuel economy improvement, compared to the production controller. Note that in the "Errands" driving profile, the electrical load demand is higher than for the EPA cycle, favoring the A-PMP strategy in increasing the fuel savings.

Finally, as Tables 7-8 show, the A-PMP control produces very similar results to the optimal PMP solution, indicating that the penalty introduced by the sub-optimality of the forward-looking controller is extremely limited, and amply compensated by the fuel economy benefits achieved, compared to the baseline control. 


\section{CONCLUSIONS}

This paper describes the development, implementation and experimental verification of a supervisory energy management strategy for a $12 \mathrm{~V}$ vehicle electrical system, which aims at controlling the alternator duty cycle for fuel economy while meeting the current demand from the auxiliary loads.

Starting from a validated control-oriented model of the electrical system of a passenger car, the supervisory energy management was cast as a constrained global optimal control problem and an analytical solution was found through the Pontryagin's Minimum Principle (PMP).

A thorough analysis was then conducted to evaluate the sensitivity of the PMP solution to various vehicle usage conditions, in particular studying the influence of driving cycles and electrical load current demand.

The results obtained led to the formulation of an adaptive control strategy that was eventually implemented in a rapid prototyping system and verified experimentally on a test vehicle. The Adaptive-PMP strategy consisted of applying corrections based on the average load current demand and on the tracking of a desired battery SOC. Drivability issues originating from chattering of the control output (which is common to this control design approach) were addressed in the final tuning and implementation of the strategy, and verified in vehicle.

The proposed control strategy was characterized and compared to simulation results obtained for the baseline (production) control algorithm for two different driving and load current profiles. In both cases, significant and consistent improvements in vehicle fuel economy were demonstrated, in the order of $1 \%$. While this number appears small in absolute value, it is worth noting that it was achieved at zero incremental cost, without any modification to the system hardware, and by solely modifying the alternator duty cycle control strategy.

It is worth noting that the fuel economy improvement is achieved at the expense of a higher utilization of the battery. On the other hand, the experimental results show that the magnitude of the current applied to the battery and the consequent voltage fluctuations are significantly smaller than the typical values expected during an engine cranking event, indicating that the impact on battery life might be limited.

To this extent, future work will address the potential impact on the battery life resulting from the application of the proposed control strategy.

\section{ACKNOWLEDGEMENTS}

The work presented in this paper was funded by the U.S. Department of Energy (Award DE-EE0003347). The authors are grateful to Fiat Chrysler Automobiles (FCA) for providing the information and suggestions that led to the development of this work.

\section{REFERENCES}

Chiara, F. and Canova, M. (2012). A review of energy consumption, management and recovery in automotive systems with considera- tions on future trends. Proc. Inst. Mech. Engr., Part D: Journal of Automobile Engineering.

Couch, J., Fiorentini, L., and Canova, M. (2013). An ecms-based approach for the energy management of a vehicle electrical system. IFAC Symposium on Advances in Automotive Control.

Geering, H.P. (2007). Optimal Control with Engineering Applications. Springer.

Kessels, J., Koot, M., de Jager, B., van den Bosch, P., Aneke, N., and Kok, D. (2007). Energy management for the electric powernet in vehicles with a conventional drivetrain. IEEE Transactions on Control Systems Technology, 15(3), 494-505.

Kim, N., Cha, S., and Peng, H. (2011). Optimal control of hybrid electric vehicles based on pontryagin's minimum principle. Control Systems Technology, IEEE Transactions on, 19(5), 1279-1287.

Koot, M., Kessels, J.T., de Jager, B., Heemels, W., Van den Bosch, P., and Steinbuch, M. (2005). Energy management strategies for vehicular electric power systems. Vehicular Technology, IEEE Transactions on, 54(3), 771-782.

Lyu, M., Doo, B., and $\mathrm{Ku}$, Y. (2007). A study of vehicle fuel economy improvement potential by optimization of the cooling and ancillary systems of a heavy duty engine. SAE Technical Paper 2007-01-1772.

Onori, S., Serrao, L., and Rizzoni, G. (2010). Adaptive equivalent consumption minimization strategy for hybrid electric vehicles.

Paganelli, G., Ercole, G., Brahma, A., Guezennec, Y., and Rizzoni, G. (2000a). A general formulation for the instantaneous control of the power split in charge-sustaining hybrid electric vehicles. In Proceedings of 5th Intl Symposium on Advanced Vehicle Control, Ann Arbor, $M I$

Paganelli, G., Guerra, T., Delprat, S., Santin, J., Delhom, M., and Combes, E. (2000b). Simulation and assessment of power control strategies for a parallel hybrid car. Proceedings of the Institution of Mechanical Engineers, Part D: Journal of Automobile Engineering, 214(7), 705-717.

Pisu, P. and Rizzoni, G. (2007). A comparative study of supervisory control strategies for hybrid electric vehicles. Control Systems Technology, IEEE Transactions on, 15(3), 506-518.

Sciarretta, A., Back, M., and Guzzella, L. (2004). Optimal control of parallel hybrid electric vehicles. Control Systems Technology, IEEE Transactions on, 12(3), 352-363.

Serrao, L., Onori, S., and Rizzoni, G. (2011). A comparative analysis of energy management strategies for hybrid electric vehicles. $133(3)$.

Serrao, L., Onori, S., and Rizzoni, G. (2009). Ecms as a realization of pontryagin's minimum principle for hev control.

Shen, J., Masrur, A., Garg, V., and Monroe, J. (2003). Automotive electric power and energy management-a system approach. Business Briefing: Global Automotive Manufacturing and Technology.

Silva, C., Ross, M., and Farias, T. (2009). Analysis and simulation of low-cost strategies to reduce fuel consumption and emissions in conventional gasoline light-duty vehicles. Energy Conversion and Management, 50(2), 215-222.

Stockar, S., Marano, V., Canova, M., Rizzoni, G., and Guzzella, L. (2011). Energy-optimal control of plug-in hybrid electric vehicles for real-world driving cycles. IEEE Transactions on Vehicular Technology, 60(7), 2949-2962.

Stockar, S., Marano, V., Rizzoni, G., and Guzzella, L. (2010). Optimal control for plug-in hybrid electric vehicle applications. In American Control Conference (ACC), 2010, 5024-5030. IEEE. 